\title{
Note on single-field inflation and the swampland criteria
}

\author{
Suratna Das* \\ Department of Physics, Indian Institute of Technology, Kanpur 208016, India
}

(Received 21 September 2018; published 9 April 2019)

\begin{abstract}
The recently proposed swampland criteria aim to evade any (meta-) stable de Sitter constructions within string landscapes, making it difficult to accommodate accelerating phases, like dark energy domination and inflationary epoch, in cosmology. In this note, we analyze the status of various models of single-field slowroll inflation given the old as well as the refined swampland conjectures, which constrain the form of scalar potentials in any low-energy effective field theory residing in the landscapes. In particular, we note that warm inflation turns out to be the most befitting scenario as long as lifting the tensions with swampland criteria is concerned.
\end{abstract}

DOI: 10.1103/PhysRevD.99.083510

Despite the remarkable agreement with the current data, it seems that the cosmological inflationary paradigm has yet to pass a few litmus tests, some of which are age-old issues $^{1}$ and some of which have been coined very recently, dubbed the swampland criteria. These swampland criteria, initially proposed in [6] and later refined in [7], caused enough ruckus in the scientific community, as these criteria, if proved to be true, can potentially jeopardize our present understanding of the Universe, both present as well as early (exponential expansions), as has been claimed in [8].

String theory, probably the best-known theory of quantum gravity to date, provides us with a vast "landscape," with nearly $10^{500}$ vacua, where consistent quantum theory of gravity is believed to be formulated with consistent lowenergy effective field theories (EFTs). But such landscapes are known to be surrounded by even larger regions, dubbed swamplands, where apparently consistent EFTs, which are coupled to gravity, are, in reality, inconsistent with the quantum theory of gravity. Hence, it is desirable for consistent EFTs not to lie in the swamplands, which eventually has led us to some set of conjectures, such as the weak gravity conjecture proposed a decade ago [9], and another set of swampland criteria that has been proposed quite recently [6,7]. It has been noted that though the Minkowski and anti-de Sitter vacua can be easily obtained in string landscapes, it is a taxing job to embed de Sitter vacua in these coveted regions [10]. Such observations

\footnotetext{
*suratna@iitk.ac.in

${ }^{1}$ Such as the initial condition problem [1-3] and the quantumto-classical transition of primordial perturbations $[4,5]$.

Published by the American Physical Society under the terms of the Creative Commons Attribution 4.0 International license. Further distribution of this work must maintain attribution to the author(s) and the published article's title, journal citation, and DOI. Funded by SCOAP ${ }^{3}$.
}

have led to the belief that de Sitter vacua rather reside in the swamplands, which makes the case difficult for the theories or paradigms relying on de Sitter solutions.

The simplest class of inflationary paradigm, known as the single-field slow-roll models, depicts an early phase of (nearly) de Sitter evolution of the Universe, governed by a dynamical scalar field (known as the inflaton field) slowly rolling down its nearly flat potential. It is desirable to put the inflationary models in a UV complete field theory, where the low-energy EFT leads to inflation. It is thus obvious that not being able to obey the above-mentioned set of conjectures, dubbed the swampland criteria, instantly poses threats to the inflationary paradigm. Conversely, not being able to accommodate the observationally supported inflationary paradigm within string landscapes can also pose a threat to string theory constructions. Hence, it is an important task to recheck the status of the inflationary paradigm given the string swampland criteria $[6,7]$.

The first set of swampland criteria was proposed in [6] which were as follows:

(i) Swampland criterion I (the distance conjecture): This criterion limits the range traversed by scalar fields in field space as

$$
\frac{|\Delta \phi|}{M_{\mathrm{Pl}}} \lesssim \Delta
$$

(ii) Swampland criterion II (the de Sitter Conjecture): This criterion limits the gradient of scalar potentials in an EFT as

$$
M_{\mathrm{Pl}} \frac{\left|V_{\phi}\right|}{V} \gtrsim c
$$

where $M_{\mathrm{Pl}}$ is the reduced Planck mass, and $V_{\phi} \equiv d V / d \phi$. Here $\Delta, c \sim \mathcal{O}(1)$. The actual value of $c$, which is a constant, depends on the details of compactification and 
often turns out to be greater than $\sqrt{2}$ [6]. It can be readily seen why the second swampland criterion is of instant threat to the inflationary paradigm. The slow-roll parameter $\epsilon$ of the inflaton field, which is a measure of the slope of the potential, is defined as

$$
\epsilon=\frac{1}{2} M_{\mathrm{Pl}}^{2}\left(\frac{V_{\phi}}{V}\right)^{2},
$$

and $\epsilon<1$ ensures the slow roll of the inflaton field during inflation. Thus the slow-roll condition is in direct conflict with swampland criterion II.

However, demanding that no critical de Sitter vacuum exists allows one to consider values of $c$ smaller than unity, until it is positive. Thus one can even consider $c \sim \mathcal{O}\left(10^{-1}\right)$, which is as good as $c \sim \mathcal{O}(1)$ [11]. Even this seems to be not in accordance with the current observation of tensor-to-scalar ratio $(r)$. Single-field slow-roll models with canonical kinetic term give rise to both tensor and scalar perturbations with a ratio of their amplitudes as

$$
r=16 \epsilon .
$$

This yields

$$
r=16 \epsilon \gtrsim 8 c^{2} \sim 0.08
$$

considering $c \sim 10^{-1}$. Observations by PLANCK satellite and BICEP2/KEK Array ground-based small aperture telescopes together put an upper bound on this tensor-toscalar ratio $r \lesssim 0.064$ [12]. Thus apparently swampland criterion II is in direct conflict with the current data as has been pointed out in [8]. The lower the upper bound on $r$ to be set by the future observations, the more the tension with the criterion would become. This realization has led to a flurry of papers where several single-field models have been analyzed just to conclude that they are indeed inconsistent with the swampland criterion II $[8,11,13-16]{ }^{2}$

However, it was also readily observed that by going beyond the single-field domain, the multifield models of inflation can be accommodated with the swampland criterion in question. In [11], the authors considered curvaton models $[17,18]$, wherein the primordial perturbations are generated by a different scalar field, called the curvaton, at the end of inflation when the curvaton isocurvature perturbations get converted into curvature perturbations, and the single-field consistency relation, given in Eq. (4), does not hold in such models [19]. Hence, such models are not constrained by the relation given in Eq. (4) and the observation of $r$ is irrelevant to the

\footnotetext{
${ }^{2}$ It is noteworthy that swampland criterion $\mathrm{I}$ is not in direct tension with the observations, as writing $\Delta \phi \sim 30 \sqrt{r / 2} M_{\mathrm{Pl}}$ $[11,13]$, we see that the factor $30 \sqrt{r / 2}$ is $\mathcal{O}(1)$.
}

validity of swampland criterion II. It is observed in [13] that in the multifield model of inflation the relation between $r$ and $\epsilon$ turns out to be

$$
r=16 \epsilon c_{s}
$$

where $c_{s}$ is the speed of sound at which the curvature perturbations propagate in a multifield model. This $c_{s}$ can be written as

$$
c_{s}=\left(1+\frac{4 \Omega^{2}}{M_{\mathrm{Pl}}^{2}}\right)^{-1 / 2},
$$

where $\Omega$ is the rate of turning of the inflationary trajectory in the multifield space. As $c_{s}$ is less than unity (or at best 1), this gives $r<16 \epsilon(r=16 \epsilon)$, thus lifting the tension between the criterion given in Eq. (2) and the observational upper bound on $r$ for cases with $c_{s}<0.8$.

Hence certainly, one way to tackle swampland criterion II is by going beyond the simplest single-field models. And yet, as the simplest single-field models are the ones most favored by the data it calls for seeking single-field scenarios that would be in accordance with swampland criterion II. Recently it was proposed in [20] that non-Bunch-Davies initial state for cosmological perturbation yields [21,22]

$$
r=16 \epsilon \gamma,
$$

where the factor $\gamma$ turns out to be the ratio of the Bogoliubov transformations of the tensor and scalar perturbations,

$$
\gamma=\frac{\left|\alpha_{k}^{(t)}+\beta_{k}^{(t)}\right|^{2}}{\left|\alpha_{k}^{(s)}+\beta_{k}^{(s)}\right|^{2}},
$$

where $\gamma$ can be made less than unity by choosing some proper inflationary model. Thus such models featuring nonBunch-Davies vacuum can make the single-field scenario consistent with swampland criterion II. This claim has been counteracted in a recent analysis [23] where it was shown that such non-Bunch-Davies vacuum for scalar perturbations would yield large local non-Gaussianities, making them incompatible with present observations. On the other hand, considering non-Bunch-Davies vacuum only for tensor modes can make such models be in tune with both swampland criteria and current bounds on primordial nonGaussianities, as that would generate large flattened nonGaussianities only in the tensor sector [23].

The case of eternal inflation [24,25] has also been discussed in the literature [26,27] as a case of single-field inflation in the context of swampland criteria. It is well known that eternal inflation, where inflaton quantum fluctuations dominate over the classical dynamics of the inflaton field, cannot yield the observed primordial scalar spectrum as it generates way too large a scalar amplitude 
$\left[\mathcal{O}(1)\right.$, where the observed spectrum is $\left.\sim \mathcal{O}\left(10^{-9}\right)\right]$, and thus should be followed by a phase of standard slow-roll inflation. Even though eternal inflation helps explain the initial condition for slow-roll inflation and also helps populating the large number of string landscape vacua, one of which corresponds to our own Universe. As the swampland criteria are universal within a given EFT, it is thus an important task to investigate whether eternal inflation can be realized within string landscapes, though it is not bounded by the cosmological observations. As has been discussed in both these works [26,27], eternal inflation can only be accommodated in landscapes if $c \lesssim \mathcal{O}\left(10^{-2}\right)$, demanding that during eternal chaotic inflation the potential should be below Planck scale [26] and demanding a quantum jump timescale to be less than the inverse of the Hubble parameter during eternal inflation in a steep potential [27].

The purpose of this article is to point out a couple of other single-field scenarios that accommodate swampland criterion II nicely. One can easily note from Eqs. (6) and (8) that it is the suppression factor $c_{s}$ or $\gamma$ that is playing the trick to make either a multi- or a single-field model compatible with the swampland criterion. Hence, one can seek similar suppression factors arising in single-field scenarios that can make the respective models compatible with swampland criterion II. The two such scenarios are as follows:

(i) $k$ inflation: The obvious choice, in such a case, would be the $k$-inflation scenario [28] where the curvature perturbations travel with subluminal speed of sound due to noncanonical kinetic terms of the inflaton field, yielding

$$
r=16 \epsilon c_{s}
$$

again, with $c_{s}<1$. This has also been noted in [16] while discussing the case of Dirac-Born-Infeld inflation.

(ii) Warm inflation: the warm inflation [29] scenario is even more interesting as far as handling swampland criterion II is concerned, as this scenario is capable of tackling the situation in three different ways, as we state below.

Warm inflation is an alternative scenario to the generic cold inflation scenario, where the inflaton field dissipates to a thermal bath while inflating and thus maintains a constant radiation energy density throughout inflation despite the exponential expansion. Thus such a scenario does not require calling for a reheating phase at the end of inflation. In the standard cold inflation scenario an inflaton field is in need of a minimum of the potential where the field would roll down at the end of inflation to reheat the Universe by oscillating at the bottom of the potential and dissipating its energy to other fields. According to swampland criterion II, scalar fields should not have any such minima in their potentials. As warm inflation does not call for a reheating phase, it naturally is not in need of any such minima of the inflaton potential, which is rather essential in a cold inflationary setup.

Without going into the details of model building of the warm inflation scenario, one can simply write down the dissipative equation of motion of the inflaton field as

$$
\ddot{\phi}+3 H \dot{\phi}+\Gamma \dot{\phi}+V_{\phi}=0,
$$

where $\Gamma$ is the dissipative coefficient that amounts to an extra friction term in the equation of the inflaton field. Defining

$$
Q=\frac{\Gamma}{3 H},
$$

the slow roll of the inflaton field is achieved when the following criteria are satisfied [30]:

$$
\begin{gathered}
\epsilon=\frac{M_{\mathrm{Pl}}^{2}\left(\frac{V_{\phi}}{V}\right)^{2} \ll 1+Q,}{2} \ll M_{\mathrm{Pl}}^{2}\left(\frac{\left|V_{\phi \phi}\right|}{V}\right) \ll 1+Q, \\
\sigma=M_{\mathrm{Pl}}^{2}\left(\frac{V_{\phi}}{\phi V}\right) \ll 1+Q, \\
\beta=M_{\mathrm{Pl}}^{2}\left(\frac{\Gamma_{\phi} V_{\phi}}{\Gamma V}\right) \ll 1+Q .
\end{gathered}
$$

Thus it can be easily seen that $\epsilon<1$ is no longer the criterium to be met to yield the slow roll of the inflaton field. Above all, in the strong dissipative regime, when $\Gamma>3 H$ and $Q>1$, then even with $c \sim \mathcal{O}(1)$ swampland criterion II would not meddle with the slow rolling of the inflaton field. This is a definite advantage of warm inflation over all the other scenarios discussed so far in the context of swampland criteria.

Besides, the warm inflation scenario yields a tensor-to-scalar ratio as [30]

$$
r=\left(\frac{H}{T}\right) \frac{16 \epsilon}{(1+Q)^{5 / 2}},
$$

where $T$ is the temperature of the thermal bath with $T>H$ (and $Q$ is, of course, positive). Hence, it can be easily seen that one of the features of warm inflation is $r<16 \epsilon$, which is in favor of swampland criterion II as far as the cosmological observations are concerned. 
As a passing comment, the warm inflationary scenario can also be successfully realized in a large class of string theory models [31-33]. ${ }^{3}$

After the refinement: It was later pointed out in [14] that in order to constrain de Sitter/inflating vacua in string theory, which has given rise to the two swampland criteria we have been discussing so far, one should rather restrict slow roll altogether. Swampland criterion II, or the de Sitter conjecture, does indeed attempt to do that by demanding $\epsilon>1$. But slow roll depends on two slow-roll parameters $\epsilon$ and $\eta\left(\equiv M_{\mathrm{Pl}}^{2} V_{\phi \phi} / V\right)$, where one requires $\epsilon \ll 1$ and $|\eta| \ll 1$ for slow roll. Hence in order to restrict slow roll either of the two slow-roll parameters should be greater than 1 .

Followed by this argument of refining swampland criterion II based on slow-roll arguments [14], a refined version of the de Sitter conjecture has now been proposed in [7], where it was shown that the refined swampland criterion II directly follows from implementing swampland criterion I or the distance conjecture, which has been more firmly established in many string theory constructions. The distance conjecture suggests that scalar fields (moduli) traveling to large (trans-Planckian) geodesic distances give rise to a tower of light states with masses $m \sim e^{-a \Delta \phi}$, where $\Delta \phi$ is the change in the field value in Planck units and $a \sim \mathcal{O}(1)$. If the accelerating universe has a causal region with an apparent horizon of radius $R$, then the number of effective degrees of freedom, increased by having these towers of light particles with exponentially small masses, increases the entropy within the causally connected region. This increased entropy then influences how the scalar potential behaves in any weak coupling limit. In order to see the effect on the potential, the effective number of particle species (below the cutoff of the effective theory) and entropy coming from the towers of particles were parametrized as [7]

$$
N(\phi)=n(\phi) e^{b \phi}, \quad S_{\text {tower }}(N, R) \sim N^{\gamma} R^{\delta},
$$

\footnotetext{
${ }^{3}$ After this analysis was presented, which points out several advantages of warm inflation over the standard cold inflation given the swampland criteria, two more analyses of warm inflation in the light of swampland have been done, one by Motaharfar et al. [34] and the other by the author of this manuscript [35] analyzing the parameter space of warm inflation to best fit the swampland criteria. Motaharfar et al. [34], without relating with the observational upper bound on $r$, concludes that taking both the swampland criteria together would drive warm inflation to take place deep into the strong dissipative regime where $Q$ should be larger, at least of the order of the minimum number of $e$-folds required. Such strong dissipative regime warm inflation scenarios can yield scale depend scalar power spectrum which would contradict the observations. On the other hand, the analysis made in [35] shows that treating the two swampland criteria separately with the observational upper bound on $r$ actually requires $1+Q$ to be slightly greater than unity. Such a weak dissipative regime $(Q \leq 1)$ of warm inflation is more in accordance with current observations.
}

respectively, where $n(\phi)$ is the effective number of towers of states that are becoming light and $b$ depends on the mass gaps and other features of the towers and is often different from $a$. However, the Bousso bound [36] suggests that $S_{\text {tower }} \leq R^{2}$ and the distance conjecture suggests that $n(\phi)$ should increase monotonically as $\phi$ increases. Thus demanding both $S_{\text {tower }} \leq R^{2}$ and $d n(\phi) / d \phi>0$ and knowing that for de Sitter horizon $R^{2} \sim H^{-2} \sim V^{-1}$, one arrives at the condition

$$
M_{\mathrm{Pl}} \frac{\left|V_{\phi}\right|}{V}>\frac{2 b \gamma}{\delta-2} \equiv c
$$

which is the old de Sitter conjecture or swampland criterion II and violates the slow-roll condition $\epsilon<1$. On the other hand, to have stable accelerating vacua, the semiclassical picture should not break down due to large quantum corrections of $\phi$. It can be seen from the equation of motion of the quantum modes of the scalar field that the quantum modes become tachyonic on horizon crossing if $V_{\phi \phi}$ becomes less than $-c^{\prime} H^{2} \sim-c^{\prime} / R^{2} \sim-c^{\prime} V$. Thus a stable semiclassical picture would call for $M_{\mathrm{Pl}}^{2} V_{\phi \phi} \geq-c^{\prime} V$ with $c^{\prime} \sim \mathcal{O}(1)$, which implies that the condition $V_{\phi \phi} \leq$ $-c^{\prime} V$ would evade any (meta-) stable de Sitter vacua. Thus the refined de Sitter conjecture is now read as

$$
M_{\mathrm{Pl}} \frac{\left|V_{\phi}\right|}{V}>c \quad \text { or } \quad M_{\mathrm{Pl}}^{2} \frac{\min \left(V_{\phi_{i} \phi_{j}}\right)}{V} \leq-c^{\prime}
$$

where both $c$ and $c^{\prime}$ are of order unity, which also violates the slow-roll conditions. Thus the refined de Sitter conjecture restricts the form of the scalar potential within a causal region (in a weak coupling regime).

The above conditions can be written in terms of slow-roll parameters within the framework of single-field inflation as

$$
\epsilon>\frac{c^{2}}{2} \quad \text { or } \quad \eta \leq-c^{\prime}
$$

We have been discussing the first condition, i.e., $\epsilon>c^{2} / 2$ (in which case $\eta$ can be greater than $-c^{\prime}$ ), so far, and have noticed that it is quite difficult to accommodate single-field inflation in string landscapes given these criteria, except for a few cases like non-Bunch-Davies vacuum for tensor modes, $k$ inflation, and warm inflation. It is noteworthy that though the refined de Sitter conjecture puts bound on the form of the potential and hence restricts the slow-roll dynamics, the arguments, as presented in [7] and as has been illustrated above, depends upon the size of the causal horizon $R$ and the entropy inside it which is restricted by the Bousso bound [36]. For $k$ inflation the scalar modes travel with a speed $c_{s}<1$, and thus have a sound horizon $\left(c_{s} H^{-1}\right)$ within which they are causally connected [28]. Thus apparently the refined de Sitter conjectures as well as the Bousso bound (which depends on the size of the causal 
region) might take a different form in such scenarios, and we defer this analysis for a future study.

We now turn towards the second criteria $\eta \leq-c^{\prime}$ with $\epsilon \ll 1$, which helps evade the contradiction coming from the observed upper bound on $r$ (which demands $\epsilon<0.004)$. First of all, this option implies that the inflaton potential must be concave. But $\eta$ is directly related to another primordial observable, namely the scalar spectral index, which for the single-field model turns out to be

$$
n_{s}-1=2 \eta-6 \epsilon,
$$

and for $\epsilon \ll 1$, this would be $n_{s}-1 \approx 2 \eta$. The scalar spectral tilt is observationally a well-constrained primordial parameter and according to the recent Planck observations $n_{s}=0.9649 \pm 0.0042$ at $68 \%$ C.L. [12]. Hence, demanding that $|\eta| \geq \mathcal{O}(1)$, to be in tune with the refined de Sitter criterion, one faces direct contradiction with the current measurement of the scalar spectral tilt [37-39]. This option of the refined de Sitter conjecture thus turns out to be even more difficult to accommodate than the previously stated de Sitter conjecture $\left(\epsilon>c^{2} / 2\right)$ as far as single-field inflation is concerned. It was even emphasized in [39] that single-field inflation cannot be accommodated in the string landscapes, given the refined swampland criteria, without fine-tuning one of the parameters $c$ and $c^{\prime}$, for both of which the refined criteria claim to be of order unity. Thus, even treating both these conditions, appearing in refined de Sitter conjecture [Eq. (18)], separately, one runs into contradiction with observations as well as with the criteria themselves, unless one fine-tunes the parameters $c$ or $c^{\prime}$ to at least 2 orders lower than unity. Other single-field scenarios studied in this context, like Higgs inflation [40], type I hilltop inflation [41], and minimal gauge inflation [42], also suggest finetuning of both or either of the parameters to accommodate the respective scenarios within string landscapes.

The option $\eta \leq-c^{\prime}$ with $\epsilon \ll 1$ also makes the curvaton model run into a conflict with observations as for such models one has

$$
n_{s}-1=2 \frac{\dot{H}}{H^{2}}+2 \eta_{\sigma \sigma}
$$

where $\sigma$ is the curvaton field, and with $\epsilon \sim-\dot{H} / H^{2} \ll 1$, one gets $n_{s}-1 \approx 2 \eta_{\sigma \sigma}$, which again to meet the de Sitter conjecture should be of order unity [37]. Thus curvaton models perform better under the condition $\epsilon>c^{2} / 2$. However, a recent analysis [43] shows that this option, $\eta \leq-c^{\prime}$ with $\epsilon \ll 1$, can make hilltop eternal inflation in accordance with the refined swampland criteria, where one quantum jump timescale exceeds the expansion rate of the Universe during eternal inflation if $\eta>-\sqrt{3}$, which is marginally in tune with the alternative option provided by the refined de Sitter conjecture. But, as is it known, to explain the observations, eternal inflation should be followed by a slow-roll phase, in which case warm inflation turns out to be a better option as has been pointed out in [43].

Let us now judge the option $\eta \leq-c^{\prime}$ with $\epsilon \ll 1$ in the realm of the warm inflation scenario. The scalar spectral tilt in warm inflation turns out to be [44]

$$
n_{s}-1=\frac{1}{Q}\left(-\frac{9}{4} \epsilon+\frac{3}{2} \eta-\frac{9}{4} \beta\right)
$$

where the slow-roll parameter $\beta$ is nonzero if the inflaton decay-width $\Gamma$ depends on inflaton field $\phi$. With $\epsilon \ll 1$, the above equation becomes

$$
n_{s}-1 \approx \frac{3}{4 Q}(2 \eta-3 \beta) .
$$

Since $\eta$ is negative and $|\eta|$ is of order unity as per the refined swampland criterion, we see that $Q$ should be $\mathcal{O}(10)$ (irrespective of $\beta$ being identically 0 or turning out to be of order unity) in order to keep warm inflation in accordance with observed scalar spectral tilt. Hence, we note that both the de Sitter options can be accommodated in the warm inflation scenario, though the first option $\epsilon>c^{2} / 2$ turns out to be more preferable as it does not demand $Q$ to be of $\mathcal{O}(10)$ [35].

In conclusion, we point out that, despite the recent folklore that the single-field models are in tension with the string swampland criterion $[8,11,13-16]$, there are at least two single-field scenarios, such as non-Bunch-Davies initial condition for tensor modes [23] and warm inflation $[29,30]$, that evade the apparent discrepancies to make themselves compatible with those criteria (even after the proposed refined version of the criteria [7]). Warm inflation, in particular, turns out to be more interesting as such a scenario will survive even if the constant $c$ and $c^{\prime}$ appearing in swampland criterion II turn out to be of $\mathcal{O}(1)$ in future, which is not the case for any other single-field slow-roll model. All in all, the single-field scenario can potentially pass the litmus test of swampland criteria with flying colors, provided we warm it up a bit.

\section{ACKNOWLEDGMENTS}

The work of S. D. is supported by the Department of Science and Technology, Government of India under Grant No. IFA13-PH-77 (INSPIRE Faculty Award). The author thanks Arjun Bagchi for useful discussions. 
[1] A. Ijjas, P. J. Steinhardt, and A. Loeb, Phys. Lett. B 723, 261 (2013).

[2] R. Brandenberger, Int. J. Mod. Phys. D 26, 1740002 (2017).

[3] S. Das and R. Rangarajan, arXiv:1805.03160.

[4] J. Martin, V. Vennin, and P. Peter, Phys. Rev. D 86, 103524 (2012).

[5] S. Das, K. Lochan, S. Sahu, and T. P. Singh, Phys. Rev. D 88, 085020 (2013); 89, 109902(E) (2014).

[6] G. Obied, H. Ooguri, L. Spodyneiko, and C. Vafa, arXiv: 1806.08362.

[7] H. Ooguri, E. Palti, G. Shiu, and C. Vafa, Phys. Lett. B 788, 180 (2019).

[8] P. Agrawal, G. Obied, P. J. Steinhardt, and C. Vafa, Phys. Lett. B 784, 271 (2018).

[9] N. Arkani-Hamed, L. Motl, A. Nicolis, and C. Vafa, J. High Energy Phys. 06 (2007) 060.

[10] T. D. Brennan, F. Carta, and C. Vafa, Proc. Sci. TASI2017 (2017) 015.

[11] A. Kehagias and A. Riotto, Fortschr. Phys. 66, 1800052 (2018).

[12] Y. Akrami et al. (Planck Collaboration), arXiv:1807.06211.

[13] A. Achúcarro and G. A. Palma, J. Cosmol. Astropart. Phys. 02 (2019) 041.

[14] S. K. Garg and C. Krishnan, arXiv:1807.05193.

[15] M. Dias, J. Frazer, A. Retolaza, and A. Westphal, Fortschr. Phys. 67, 1800063 (2019).

[16] W. H. Kinney, S. Vagnozzi, and L. Visinelli, arXiv:1808 .06424 .

[17] D. H. Lyth and D. Wands, Phys. Lett. B 524, 5 (2002).

[18] D. H. Lyth, C. Ungarelli, and D. Wands, Phys. Rev. D 67, 023503 (2003).

[19] C. T. Byrnes, M. Cortês, and A. R. Liddle, Phys. Rev. D 90, 023523 (2014).

[20] S. Brahma and M. W. Hossain, J. High Energy Phys. 03 (2019) 006.
[21] A. Ashoorioon, K. Dimopoulos, M. M. Sheikh-Jabbari, and G. Shiu, J. Cosmol. Astropart. Phys. 02 (2014) 025.

[22] A. Ashoorioon, K. Dimopoulos, M. M. Sheikh-Jabbari, and G. Shiu, Phys. Lett. B 737, 98 (2014).

[23] A. Ashoorioon, Phys. Lett. B 790, 568 (2019).

[24] A. Vilenkin, Phys. Rev. D 27, 2848 (1983).

[25] A. H. Guth, J. Phys. A 40, 6811 (2007).

[26] H. Matsui and F. Takahashi, Phys. Rev. D 99, 023533 (2019).

[27] K. Dimopoulos, Phys. Rev. D 98, 123516 (2018).

[28] J. Garriga and V. F. Mukhanov, Phys. Lett. B 458, 219 (1999).

[29] A. Berera, Phys. Rev. Lett. 75, 3218 (1995).

[30] S. Bartrum, A. Berera, and J. G. Rosa, J. Cosmol. Astropart. Phys. 06 (2013) 025.

[31] M. Bastero-Gil, A. Berera, J. B. Dent, and T. W. Kephart, arXiv:0904.2195.

[32] M. Bastero-Gil, A. Berera, and J. G. Rosa, Phys. Rev. D 84, 103503 (2011).

[33] Y.-F. Cai, J. B. Dent, and D. A. Easson, Phys. Rev. D 83, 101301 (2011).

[34] M. Motaharfar, V. Kamali, and R. O. Ramos, Phys. Rev. D 99, 063513 (2019).

[35] S. Das, Phys. Rev. D 99, 063514 (2019).

[36] R. Bousso, J. High Energy Phys. 07 (1999) 004.

[37] H. Fukuda, R. Saito, S. Shirai, and M. Yamazaki, arXiv: 1810.06532.

[38] P. Agrawal and G. Obied, arXiv:1811.00554.

[39] C.-I. Chiang, J. M. Leedom, and H. Murayama, arXiv: 1811.01987.

[40] D. Y. Cheong, S. M. Lee, and S. C. Park, Phys. Lett. B 789, 336 (2019).

[41] C.-M. Lin, Phys. Rev. D 99, 023519 (2019).

[42] S. C. Park, J. Cosmol. Astropart. Phys. 01 (2019) 053.

[43] W. H. Kinney, Phys. Rev. Lett. 122, 081302 (2019).

[44] L. M. H. Hall, I. G. Moss, and A. Berera, Phys. Rev. D 69 , 083525 (2004). 